\title{
Robustness of Light-Transport Processes to Bending Deformations in Graded-Index Multimode Waveguides
}

\author{
Dirk E. Boonzajer Flaes, ${ }^{1}$ Jan Stopka, ${ }^{3,2}$ Sergey Turtaev, ${ }^{4,5}$ Johannes F. de Boer, ${ }^{1}$ \\ Tomáš Tyc, ${ }^{3,2, *}$ and Tomáš Čižmár ${ }^{2,4,5}$ \\ ${ }^{1}$ LaserLaB Amsterdam, Department of Physics and Astronomy, VU University Amsterdam, \\ De Boelelaan 1081, 1081 HV Amsterdam, The Netherlands \\ ${ }^{2}$ Institute of Scientific Instruments of CAS, Královopolská 147, 61264 Brno, Czech Republic \\ ${ }^{3}$ Department of Theoretical Physics and Astrophysics, Masaryk University, Kotlářská 2, 61137 Brno, Czechia \\ ${ }^{4}$ Leibniz Institute of Photonic Technology, Albert-Einstein-Straße 9, 07745 Jena, Germany \\ ${ }^{5}$ School of Science and Engineering, University of Dundee, \\ Ewing building, Nethergate, DDI 4HN Dundee, Scotland, United Kingdom
}

(Received 6 December 2017; published 6 June 2018)

\begin{abstract}
Light transport through a multimode optical waveguide undergoes changes when subjected to bending deformations. We show that optical waveguides with a perfectly parabolic refractive index profile are almost immune to bending, conserving the structure of propagation-invariant modes. Moreover, we show that changes to the transmission matrix of parabolic-index fibers due to bending can be expressed with only two free parameters, regardless of how complex a particular deformation is. We provide detailed analysis of experimentally measured transmission matrices of a commercially available graded-index fiber as well as a gradient-index rod lens featuring a very faithful parabolic refractive index profile. Although parabolicindex fibers with a sufficiently precise refractive index profile are not within our reach, we show that imaging performance with standard commercially available graded-index fibers is significantly less influenced by bending deformations than step-index types under the same conditions. Our work thus predicts that the availability of ultraprecise parabolic-index fibers will make endoscopic applications with flexible probes feasible and free from extremely elaborate computational challenges.
\end{abstract}

DOI: 10.1103/PhysRevLett.120.233901

With the availability of high-performance spatial light modulation, new imaging techniques based on holographic control of light through multimode fibers (MMFs) has emerged recently [1-3]. With extremely compact dimensions, MMF-based imaging techniques are particularly suited to various forms of endoscopy. Several techniques of endomicroscopy, especially laser-scanning-based approaches [4,5] including multiphoton excitation [6], superresolution [7], as well as wide-field techniques such as bright-field, dark-field, and light-sheet microscopy $[5,8,9]$, have already been demonstrated.

These approaches have been treating MMFs as random media $[10,11]$ and therefore they required a calibration step, in which the light transport through MMF is quantitatively analyzed. This is typically based on interferometric approaches [2] or by employing phase conjugation [12], and it consists of a sequence of measurements where the

Published by the American Physical Society under the terms of the Creative Commons Attribution 4.0 International license. Further distribution of this work must maintain attribution to the author(s) and the published article's title, journal citation, and DOI. number of measurements exceeds the number of modes allowed to propagate through the MMF. These measurements then lead to the reconstruction of a transmission matrix (TM) [2,13-15] — a linear relation between conveniently chosen representations of input and output modes containing the complete information about light transport through the MMF. During this procedure and subsequent imaging, the MMF is required to remain locked to the same position (contortion) since any deformation would introduce changes to the TM and as a result affect the imaging quality $[5,16,17]$.

In 2015, it was shown that commercially available stepindex MMFs, at length scales relevant for imaging applications, are of sufficient quality to allow for prediction of the TM based on numerical simulations [18]. Moreover, this study demonstrated that the influence of significant bending deformations can be theoretically predicted and taken into account in imaging applications. However, implementing such corrections is computationally very demanding and it requires very precise knowledge of the fiber layout.

In this work we show that employing MMFs with a perfect parabolic refractive index profile (PI) eliminates both of these problems. Unlike in step-index MMFs, bending of 
PI MMFs is not associated with cross-coupling of power between idealized propagation invariant modes (PIMs).

Moreover, the influence of bending is equivalent to a minuscule increase in fiber length to a good approximation, regardless of the radius of curvature and the length of the bent segment. The associated deformation operators describing the changes to the TM due to bending are diagonal in the representation of PIMs and commute with one another. Therefore, the order of bends in the fiber is unimportant, and the deformation operators for any threedimensional adiabatic contortion of a PI MMF can be expressed upon the knowledge of only two parameters, one describing the overall curvature, the other the overall twist. Below we introduce a rigorous theoretical model for light propagation through PI MMFs undergoing bending, which results in conservation of PIMs. We then experimentally verify conservation of PIMs using a large core parabolicindex medium with a high-quality refractive index pattern. Furthermore, we demonstrate that commercially available graded-index MMFs suffer from strong deviations from the parabolic refractive index profile. Finally, we demonstrate that even with the manufacturing imperfections, the imaging performance of graded-index MMFs is indeed significantly less affected by deformation when compared to step-index MMFs.

We consider an idealized fiber with cylindrically symmetric refractive index $n(r)$ satisfying

$$
n^{2}(r)=n_{0}^{2}\left(1-\frac{r^{2}}{b^{2}}\right)
$$

where radial coordinate $r$ extends to infinity. Here, $n_{0}$ is the refractive index on the axis and $b$ is a scaling parameter. In real fibers, $n$ becomes constant beyond a certain radius (in the cladding), which, however, affects only the highest order guided modes. Solving the scalar Helmholtz equation for a straight waveguide $[19,20]$ leads to identification of scalar PIMs as

$$
\begin{aligned}
\psi_{l, m}(r, \varphi, z)= & \sqrt{\frac{\alpha}{2 \pi} \frac{2 m !}{(m+|l|) !}} \exp \left(-\frac{\alpha r^{2}}{2}\right) \\
& \times\left(\alpha r^{2}\right)^{|l| / 2} L_{m}^{|l|}\left(\alpha r^{2}\right) e^{i l \varphi} e^{i \beta_{l, m} z} .
\end{aligned}
$$

Here, $L_{m}^{|l|}$ are associated Laguerre polynomials, $\alpha=k n_{0} / b$, $k=2 \pi / \lambda$ is the vacuum wave number, $l \in \mathbb{Z}$ is the orbital angular momentum (OAM) index of the PIM, and the index $m \in \mathbb{N}_{0}$ determines the number of radial nodes [21]. The propagation constants are

$$
\begin{aligned}
\beta_{l, m} & =\sqrt{k^{2} n_{0}^{2}-2 \alpha(|l|+2 m+1)} \\
& \approx k n_{0}-\frac{|l|+2 m+1}{b},
\end{aligned}
$$

where the approximation corresponds to the weak-guidance approximation [21].

As detailed in the Supplemental Material ([22], Sec. S1), full vectorial solution of a fiber, uniformly bent in the $x z$ plane with curvature $\rho$, leads to similar PIMs, but shifted towards the outer side of the bend, and with propagation constants modified as

$$
\begin{aligned}
\beta_{l, m, \sigma}^{\prime}= & k n_{0}\left(1+\frac{\rho^{2} b^{2}}{2}\right)-\left(\frac{1}{b}+\frac{9 \rho^{2} b}{4}\right)(|l|+2 m+1) \\
& -\frac{l \sigma+1}{2 k n_{0} b^{2}},
\end{aligned}
$$

where $\sigma= \pm 1$ for the right- and left-handed circular polarization state, respectively, and making the same weak-guidance approximation as for Eq. (3). The first term is the same for all PIMs, corresponding just to an unimportant global phase. The mode-dependent phase change is given by the other two terms. The total phase accumulated by the PIM $(l, m, \sigma)$ upon propagating the distance $L$ in the fiber is $\beta_{l, m, \sigma}^{\prime} L$; its mode-dependent part is

$$
\phi=-L\left(\frac{1}{b}+\frac{9 \rho^{2} b}{4}\right)(|l|+2 m+1)-\frac{L(l \sigma+1)}{2 k n_{0} b^{2}} .
$$

The first part of the phase is the same as if the fiber were straight but of length $L^{\prime}=L\left(1+9 \rho^{2} b^{2} / 4\right)$ instead of $L$. On the other hand, the second part corresponding to spinorbital interaction is the same as in a straight fiber of the original length $L$. To demonstrate that the effect of fiber bending is equivalent to a slight fiber stretching, we compare the phase $\phi$ with the phase $\phi^{\prime}$ of the mode with the same indices $(l, m, \sigma)$ in a straight fiber $(\rho=0)$ of length $L^{\prime}$. The difference is

$$
\phi-\phi^{\prime}=\frac{9 \rho^{2} L(l \sigma+1)}{8 k n_{0}} .
$$

This difference becomes of order of $\pi$ for fiber lengths of order of hundreds of meters for practical fibers and curvatures of order of $\mathrm{cm}^{-1}$. The effect of bending is indeed equivalent to a slight increase of the fiber length, combined with a slight shift of the center of the PIMs towards the outer side of the bend. The spin-orbital interaction is typically much larger than the effect of the bend. For strong bending (the condition is given in Ref. [22], Sec. S1), a solution in Cartesian coordinates would be required.

When the fiber is not bent uniformly but the curvature and the orientation of the curvature changes along the fiber, there may also be a torsion. Since the fiber deformation is a result of forces (or more precisely, torques) between neighboring parts of the fiber, these changes are not abrupt but smooth. Thanks to the adiabatically changing curvature, the PIMs are continuously adapting to the new bend conditions. Therefore, if we send an arbitrary PIM into 
one fiber end, its center will become shifted transversely from the fiber axis to different directions and by different amounts according to the actual fiber curvature, but its form will be retained. Therefore, the cross talk between the PIMs will stay very small. The effective length of the fiber is calculated by summing the effective lengths of infinitesimal fiber elements for which the curvature can be considered to be constant, which gives

$$
L^{\prime}=L+\frac{9 b^{2}}{4} \int_{0}^{L} \rho^{2}(z) d z
$$

In case of torsion, there will be an additional effect of geometric phase that will rotate the whole pattern by the angle corresponding to the solid angle swept by the fiber axis. For moderate curvatures, the influence of an arbitrary contortion is therefore governed by just two parameters: an effective change of fiber length and a rotation of the pattern.

Before attempting to verify the predicted behavior, we verify that the considered modes are indeed supported by the waveguide. We start with a 56-mm-long glass rod with a highly accurate parabolic index profile (PI rod), with a diameter of $350 \mu \mathrm{m}$ and a numerical aperture of 0.12 . To characterize the rod, a laser focus (circularly polarized, $1064 \mathrm{~nm}$ ) is scanned over the proximal end of the rod using a spatial light modulator (SLM). The optical field leaving the distal end is captured (by means of phase-shifting interferometry using an external reference pathway $[2,4]$ ) for every input position, schematically depicted in Fig. 1, and explained more thoroughly in Sec. S2 of Ref. [22]. The resulting field is stored in the columns of a transmission matrix. For any incident field $E_{\text {in }}$, such as a PIM, the expected output field $E_{\text {out }}$ is computed using $E_{\text {out }}=\mathrm{TM} \cdot E_{\text {in }}[10,13,23]$.

On the measured TM, we performed a basis transformation from the representation of points to the representation of PIMs, as defined by Eq. (2), shown in Fig. 1(a). As PIMs should remain conserved, this matrix should be diagonal. However, PIMs are very sensitive to alignment and mode preservation can only be expected for a perfectly aligned system. Further deviations are expected due to parameters of the rod being known with limited precision. While it is impossible to align the system with sufficient accuracy and ascertain refractive index profile parameters with sufficient precision, alignment errors and refractive index parameters can be corrected numerically through an optimization procedure (Ref. [18] and Sec. S3 of Ref. [22]).

We optimized five degrees of freedom on both rod facets, three corresponding to the spatial location of the rod and two to the incidence angle. Lastly, we optimized the steepness of the refractive index profile $(b)$, which determines the overall scaling of all PIMs.

After numerical optimization of the alignment parameters, Fig. 1(b), the TM in the PIM basis $(M)$ appears to be diagonal, indicating almost no cross talk between the

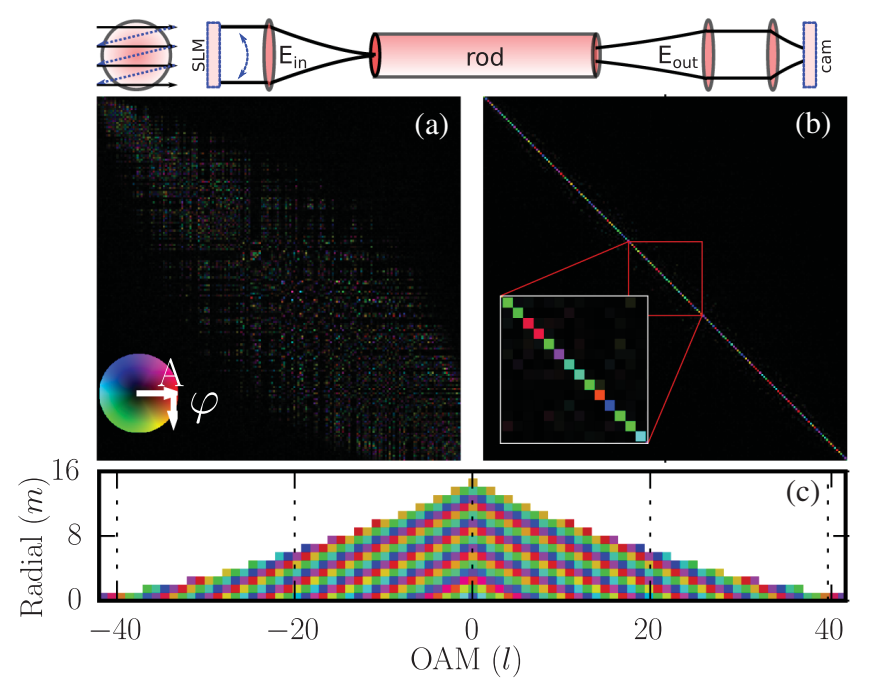

FIG. 1. Top: Diagram of transmission matrix measurement. A SLM is used to project a series of focal spots across a grid on the proximal end of the rod. The distal end output fields are measured and stored in the columns of a TM. (a) TM of the PI rod before numerical alignment, in a propagation-invariant mode (PIM) basis, ordered by $l$ and then $m$ in Eq. (2), as per Fig. 1(c) of Ref. [18]. Amplitude and phase color scale indicated in lower left. For clarity, only every third mode is displayed. (b) TM after numerical alignment. Inset shows enlarged area around the diagonal, demonstrating that only the diagonal carries significant power. (c) Measured phases and amplitudes of PIMs.

PIMs. The optimization procedure maximizes the relative power on the diagonal $P=\sum_{i}\left|M_{i i}\right|^{2} / \sum_{i, j}\left|M_{i j}\right|^{2}$. Before alignment, $P$ was only about $1.4 \%$; however, after numerical optimization, $P$ reached $95 \%$. The overall phase delay measured of all different PIMs, which we call the modal phase, is plotted in Fig. 1(c). Modes with the same combined mode number $|l|+2 m+1$ arrive with roughly the same modal phase, which is in accordance with the predicted propagation constants by Eq. (3).

Sampling all modes that are supported by the rod was unfeasible due to acquisition time. Therefore, several selection criteria were applied, as explained in Sec. S2 of Ref. [22]. Because the measured TM does not cover the full extent of the rod, some misalignment parameters can be coupled, depending on the waveguide length, which is discussed in Sec. S3 of Ref. [22].

To quantitatively evaluate the agreement between the measured and expected modal phases, the phase agreement was computed in the following way:

$$
\operatorname{PA}(L)=\left|\sum_{i} M_{i i} \exp \left(-i \beta_{i} L\right)\right|^{2} /\left(\sum_{i}\left|M_{i i}\right|\right)^{2} .
$$

Here, PA is the phase agreement, $\beta_{i}$ is the expected propagation constant for the $i$ th $l, m$ mode, and $L$ is the rod length. This quantity represents the squared ratio between the phasor sum and the amplitude sum of the modal phase 
differences. According to Eq. (3), the optimization procedure cannot optimize both $L$ and $n_{0}$ simultaneously, as their influence is indistinguishable. Therefore, only $L$ was optimized and we used a fixed value for $n_{0}$ provided by the manufacturer.

Phase agreement reached $95.8 \%$ after optimizing $L$, with residual modal phase differences shown in Fig. 2(a). They are caused by small deviations to the refractive index profile from the parabolic refractive index profile.

To estimate this deviation, we employ first order perturbation theory to compute the altered propagation speeds for a refractive index profile with a small perturbation, and optimize the shape of this perturbation on phase agreement. As a convenient basis, we chose Zernike polynomials $Z_{4}^{0}$, $Z_{6}^{0}$, and $Z_{8}^{0}$. After optimization, PA exceeds $99.3 \%$, with residual phase aberrations shown in Fig. 2(b). This results in a slightly different optimal rod length $L$ [Fig. 2(c)]. The optimized refractive index profile is shown in Fig. 2(d). We can therefore conclude that for the PI rod the model agrees very well with the observed TM.

However, PI rods do not allow bending by a significant amount. To verify our bending predictions we progressed to a graded-index MMF. These fibers typically suffer from a dip in the refractive index profile near the core axis. We measured the TM of $20 \mathrm{~mm}$ of a $50-\mu \mathrm{m}$-diameter gradedindex MMF, using the same setup but with slightly altered imaging optics (see Table S1 in Ref. [22]). The TM in PIM modes before and after alignment is shown in Figs. 3(a) and 3(b). Although the fiber is shorter, the refractive index profile is much steeper and so individual modes acquire larger phase differences [22].
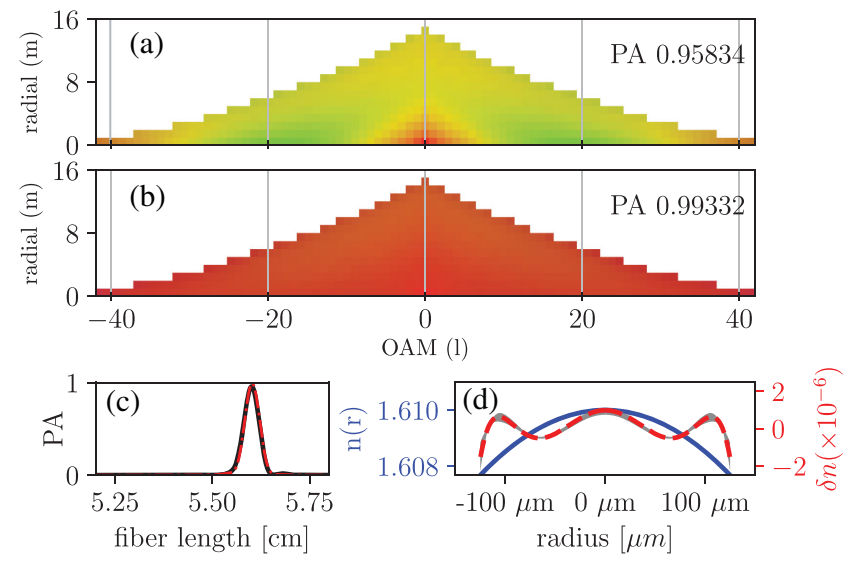

FIG. 2. (a) Modal phase difference between expected and measured PIMs after optimizing PI rod length, assuming a parabolic refractive index profile. PA is given in the figure. (b) Phase difference between expected modal phases and measured modal phases after fitting fine scale modulations to the refractive index profile. (c) PA as a function of rod length before (black) and after (red) optimizing refractive index profile. (d) Refractive index profile (blue), the detected perturbation (red), with 95\% confidence interval indicated in gray; see Sec. S3 of Ref. [22]. All color scales are the same as in Fig. 1.
The optimization procedure increased the power preserved in PIMs from 5.4\% to 53\%, significantly less than could be measured for the PI rod. Analogous modal analysis of a step-index fiber showed over $95 \%$ conservation of modal power [18]. Repeating the experiment with longer fiber lengths did not significantly improve modal preservation. In Fig. 3, lower left, some typical fiber modes reveal that output modes are severely distorted. Low order radial PIMs perform better than higher order ones, especially those with a high orbital angular momentum, even though we suspect that they suffer from fiber ellipticity. Clearly, the optical performance of a "real-life" gradedindex fiber does not match the performance of a perfectly parabolic refractive index medium. We suspect that this is caused by imperfections in the refractive index profile of the GRIN MMF.

Despite these deviations, the refractive index profile of such fibers is much closer to the parabolic distribution than step-index types, and shall exhibit better resilience to bending [24]. To verify this, we conducted a spot-scanning imaging experiment. The required input fields to scan a spot over the distal end of the fiber are measured for a straight fiber. Afterwards these input fields are projected and the total transmitted intensity through a transmissive part of a reflective sample inserted on the distal end is recorded. The fiber is then bent, but the input fields for a straight fiber are projected, repeating the imaging experiment as if no bending had taken place. Such an experiment was conducted for a step-index and two graded-index fibers with a comparable number of modes, shown in Fig. 4. This leads to severe imaging aberrations when employing the step-index MMF. However, the imaging performance is much less affected in the graded-index MMFs, where the fiber with a larger number of modes seems more bending

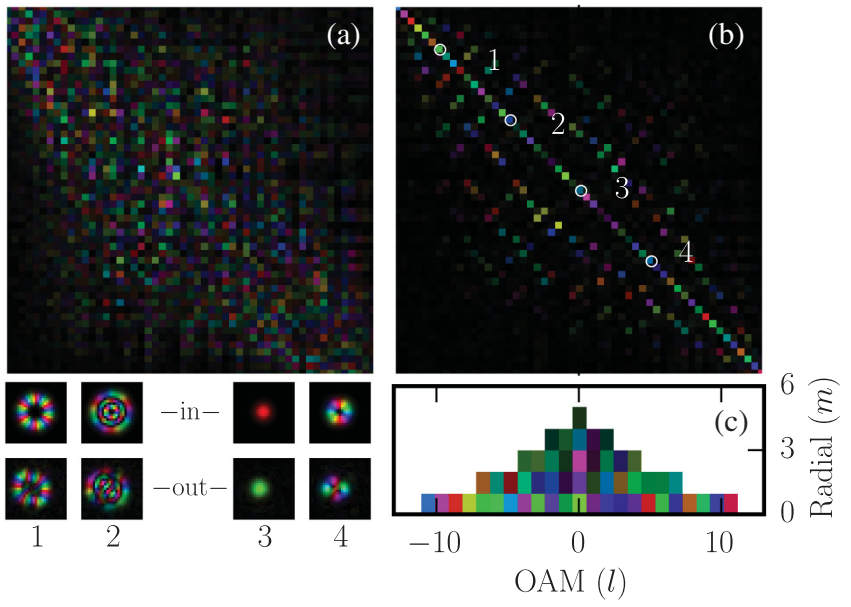

FIG. 3. Initial TM of the graded-index fiber in a PIM basis before (a) and after (b) the optimization procedure. (c) Measured phase retardations. Lower right: Predicted responses for the $(l, m)=(-6,0),(-3,2),(0,0)$, and $(2,0)$ modes at the output facet of the fiber. All color scales are the same as in Fig. 1. 


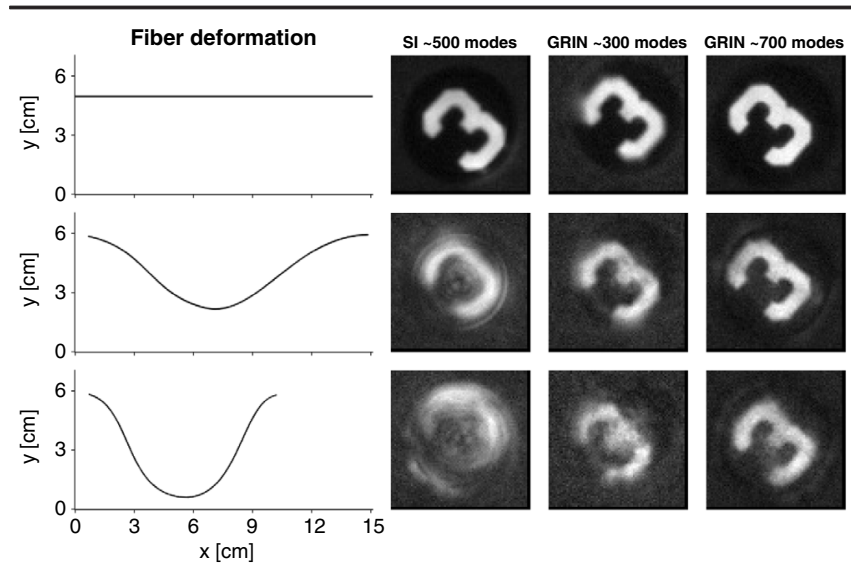

FIG. 4. Bending sensitivity of imaging performance for a stepindex (SI) MMF and two different graded-index (GRIN) MMFs. The TM is measured for a straight fiber and used to image a sample object with three different fiber layouts.

resilient. The effective length increase according to Eq. (5) is only $17 \mu \mathrm{m}$ for the tight bend, too small for a significant effect in imaging. We attribute the decrease in imaging quality to limitations of the refractive index profile. Numerical simulations reveal that the effect becomes significant for longer fiber lengths [22].

In this Letter we show that parabolic-index multimode fibers are very resilient to bending deformation. When bending curvatures are relatively small, our theoretical study indicates that all possible three-dimensional contortions can be expressed by two free parameters describing overall curvature and overall torque. The foundations of the theoretical model have been experimentally verified using a graded-index rod with a very precise refractive index profile. As this medium does not lend itself to bending deformations, we have progressed to analogous studies on commercially available fiber. These have indicated very strong deviations from the ideal parabolic refractive index profile, dramatically exceeding those of the graded-index rod as well as those observed in our previous studies in step-index fibers [18]. Since the insufficient quality of the fiber precluded a direct experimental verification of the bending resilience, we have provided a further semiempiric proof by studying the bending influence on the imaging quality achieved while using different types of fibers, clearly showing a superior performance of graded-index MMFs. This work thus demonstrates an urgent need to develop new graded-index MMFs with a higher-quality refractive index profile. This will enable numerous new possibilities in minimally invasive imaging, including highly advanced observations or optogenetic stimulation in awake and unrestrained animal models.

D. E. B. F. and J. F. de B. acknowledge support from the Netherlands Organisation for Scientific Research (NWO) with a Vici (JFdB) Grant No. 918.10.628. S. T. acknowledges funding from the EU FP7 People Programme (Marie
Curie Actions) under REA Grant Agreement No. 608144. T. T. acknowledges support of Grant No. P201/12/G028 from the Czech Science Foundation. T. T. and T. C. acknowledge support from the European Regional Development Fund through Project No. CZ.02.1.01/0.0/ 0.0/15_003/0000476. T. Č. acknowledges European Research Council (ERC), Grant No. 724530, the University of Dundee, and Scottish Universities Physics Alliance (PaLS initiative) for financial support.

*tomtyc@physics.muni.cz

[1] R. Di Leonardo and S. Bianchi, Opt. Express 19, 247 (2011).

[2] T. Čižmár and K. Dholakia, Opt. Express 19, 18871 (2011).

[3] R. N. Mahalati, R. Y. Gu, and J. M. Kahn, Opt. Express 21, 1656 (2013).

[4] S. Bianchi and R. Di Leonardo, Lab Chip 12, 635 (2012).

[5] T. Čižmár and K. Dholakia, Nat. Commun. 3, 1027 (2012).

[6] E. E. Morales-Delgado, D. Psaltis, and C. Moser, Opt. Express 23, 32158 (2015).

[7] G. P. J. Laporte, N. Stasio, C. Moser, and D. Psaltis, Opt. Express 23, 27484 (2015).

[8] Y. Choi, C. Yoon, M. Kim, T. D. Yang, C. Fang-Yen, R. R. Dasari, K. J. Lee, and W. Choi, Phys. Rev. Lett. 109, 203901 (2012).

[9] M. Plöschner, V. Kollárová, Z. Dostál, J. Nylk, T. BartonOwen, D. E. K. Ferrier, R. Chmelík, K. Dholakia, and T. Čižmár, Sci. Rep. 5, 18050 (2015).

[10] I. M. Vellekoop and A. P. Mosk, Opt. Lett. 32, 2309 (2007).

[11] I. M. Vellekoop, A. Lagendijk, and A. P. Mosk, Nat. Photonics 4, 320 (2010).

[12] I. N. Papadopoulos, S. Farahi, C. Moser, and D. Psaltis, Opt. Express 20, 10583 (2012).

[13] S. M. Popoff, G. Lerosey, R. Carminati, M. Fink, A. C. Boccara, and S. Gigan, Phys. Rev. Lett. 104, 100601 (2010).

[14] S. Popoff, G. Lerosey, M. Fink, A. C. Boccara, and S. Gigan, Nat. Commun. 1, 81 (2010).

[15] J. Carpenter, B. J. Eggleton, and J. Schröder, Opt. Express 22, 96 (2014).

[16] S. Farahi, D. Ziegler, I. N. Papadopoulos, D. Psaltis, and C. Moser, Opt. Express 21, 22504 (2013).

[17] D. Loterie, D. Psaltis, and C. Moser, Opt. Express 25, 6263 (2017).

[18] M. Plöschner, T. Tyc, and T. Čižmár, Nat. Photonics 9, 529 (2015).

[19] D. Gloge, Appl. Opt. 10, 2252 (1971).

[20] E. Snitzer, J. Opt. Soc. Am. 51, 491 (1961).

[21] A. W. Snyder and J. Love, Optical Waveguide Theory, 1st ed. (Springer, New York, 1983).

[22] See Supplemental Material at http://link.aps.org/ supplemental/10.1103/PhysRevLett.120.233901, for full details on the derivation of the vectorial solution and experimental details.

[23] J. Yoon, K. Lee, J. Park, and Y. Park, Opt. Express 23, 10158 (2015).

[24] A. M. Caravaca-Aguirre and R. Piestun, Opt. Express 25, 1656 (2017). 